\title{
Generalized Jacobi polynomials/functions and their applications
}

\author{
Ben-Yu Guo ${ }^{\mathrm{a}, 1}$, Jie Shen ${ }^{\mathrm{b}, *, 2}$, Li-Lian Wang ${ }^{\mathrm{c}, 3}$ \\ a Department of Mathematics, Shanghai Normal University, Shanghai, 200234, PR China \\ ${ }^{\mathrm{b}}$ Department of Mathematics, Purdue University, West Lafayette, IN 47907, USA \\ ${ }^{\mathrm{c}}$ Division of Mathematical Sciences, School of Physical and Mathematical Sciences, Nanyang Technological University (NTU), \\ 637371, Singapore
}

Available online 26 April 2008

\begin{abstract}
We introduce a family of generalized Jacobi polynomials/functions with indexes $\alpha, \beta \in \mathbb{R}$ which are mutually orthogonal with respect to the corresponding Jacobi weights and which inherit selected important properties of the classical Jacobi polynomials. We establish their basic approximation properties in suitably weighted Sobolev spaces. As an example of their applications, we show that the generalized Jacobi polynomials/functions, with indexes corresponding to the number of homogeneous boundary conditions in a given partial differential equation, are the natural basis functions for the spectral approximation of this partial differential equation. Moreover, the use of generalized Jacobi polynomials/functions leads to much simplified analysis, more precise error estimates and well conditioned algorithms.
\end{abstract}

(C) 2008 IMACS. Published by Elsevier B.V. All rights reserved.

MSC: 65N35; 65N22;65F05; 35J05

Keywords: Jacobi polynomials; Spectral approximation; Error estimate; High-order differential equations

\section{Introduction}

The classical Jacobi polynomials have been used extensively in mathematical analysis and practical applications (cf. [35,2,36,31]). In particular, the Legendre and Chebyshev polynomials have played an important role in spectral methods for partial differential equations (cf. [20,13,19,12,22] and the references therein). Recently, there have been renewed interests in using the Jacobi polynomials in spectral approximations, especially for problems with degenerated or singular coefficients. For instance, Bernardi and Maday [9] considered spectral approximations using the ultra-spherical polynomials in weighted Sobolev spaces. Guo [23,21,24] developed Jacobi approximations in certain Hilbert spaces with their applications to singular differential equations and some problems on infinite intervals.

\footnotetext{
* Corresponding author.

E-mail addresses: shen@math.purdue.edu (J. Shen), lilian@ntu.edu.sg (L.-L. Wang).

1 The work of this author is supported partially by NSF of China, N. 10471095, SF of Shanghai N. 04JC14062, The Fund of Chinese Education Ministry N. 20040270002, The Shanghai Leading Academic Discipline Project N. T0401, and the Fund for E-institutes of Shanghai Universities N. E03004.

2 The work of this author is partially supported by NFS grant DMS-0610646.

3 The work of this author is partially supported by the Startup Grant of NTU, Singapore MOE grant T207B2202, and Singapore NRF2007IDMIDM002-010.
} 
The Jacobi approximations were also used to obtain optimal error estimates for $p$-version of finite element methods (cf. $[3,4])$.

Recently, Shen [33] introduced an efficient spectral dual-Petrov-Galerkin method for third and higher odd-order differential equations, and pointed out that the basis functions used in [33], which are compact combinations of Legendre polynomials, can be viewed as generalized Jacobi polynomials with negative integer indexes, and their use not only simplified the numerical analysis for the spectral approximations of higher odd-order differential equations, but also led to very efficient numerical algorithms. More precisely, the resulting linear systems are well conditioned, and sparse for problems with constant coefficients. In fact, the basis functions used in [32], which are compact combinations of Legendre polynomials, can also be viewed as generalized Jacobi polynomials with indexes $\alpha, \beta \leqslant-1$. Furthermore, the special cases with $(\alpha, \beta)=(-1,0),(-1,-1)$ have also been studied in [6,21,24]. Hence, instead of developing approximation results for each particular pair of indexes, it would be very useful to carry out a systematic study on Jacobi polynomials with indexes $\alpha, \beta \leqslant-1$ which can then be directly applied to other applications. In [25], we defined the generalized Jacobi polynomials with indexes being negative integers, and presented some approximation results and applications. However, in many situations, it is helpful to define and use generalized Jacobi polynomials with arbitrary non-integer indexes. For example, when developing and analyzing Chebyshev spectral methods for boundary value problems, it becomes convenient to use generalized Jacobi polynomials with indexes $(-1 / 2-k,-1 / 2-l)$ (cf. [34]). Another example is the study of differential equations with singular coefficients of the form $(1-x)^{\alpha}(1+x)^{\beta}$.

The main purpose of this paper is to generalize the definition of the Jacobi polynomials to arbitrary indexes $\alpha, \beta \in \mathbb{R}$, and to establish their fundamental approximation results, which include, as special cases, those announced in [25] but not proved due to the page limitation of [25] as a conference proceeding paper. The main criteria that we use to define the generalized Jacobi polynomials/functions are: (i) they are mutually orthogonal with respect to the Jacobi weight, and (ii) they inherit some important properties (to be specified later) of the classical Jacobi polynomials which are essential for spectral approximations.

As an example of applications, we consider approximations of high-order differential equations with suitable boundary conditions. Mathematical modeling of some physical systems often leads to high-order differential equations. For example, high even-order differential equations often appear in astrophysics, structural mechanics and geophysics (see, e.g., [1,11]); high odd-order differential equations, such as third-order Korteweg-de-Vries (KdV) and fifth-order KdV-type equations, are routinely used in non-linear wave and non-linear optics theory (see, e.g., $[37,28,10,16,30])$.

While it is usually cumbersome to design an accurate and stable numerical algorithms using finite difference/finite element methods due to the many boundary conditions involved or using a spectral-collocation method for which special quadratures involving derivatives at the end points have to be developed (cf. [7,27,29]) or fictitious points have to be introduced [18], the spectral approximations using generalized Jacobi polynomials/functions lead to straightforward and well-conditioned implementations, and can be analyzed with a unified approach leading to more precise error estimates.

This paper is organized as follows. In the next section, we define the generalized Jacobi polynomials/functions and analyze the approximation properties of the orthogonal projection in suitably weighted Sobolev spaces. The generalized Jacobi polynomials/functions and their approximation results are used in Section 3 to construct and analyze spectral-Galerkin method for some high-order model equations. Some concluding remarks are given in the final section.

\section{Generalized Jacobi polynomials/functions}

In this section, we define the generalized Jacobi polynomials/functions (GJP/Fs), and investigate their basic properties.

We first introduce some notations. Let $\omega(x)$ be a weight function in $I:=(-1,1)$. One usually requires that $\omega \in L^{1}(I)$. However, we shall mainly concern with the cases $\omega \notin L^{1}(I)$. We shall use the weighted Sobolev spaces $H_{\omega}^{r}(I)(r=0,1,2, \ldots)$, whose inner products, norms and semi-norms are denoted by $(\cdot, \cdot)_{r, \omega},\|\cdot\|_{r, \omega}$ and $|\cdot|_{r, \omega}$, respectively. For real $r>0$, we define the space $H_{\omega}^{r}(I)$ by space interpolation. In particular, the norm and inner product 
of $L_{\omega}^{2}(I)=H_{\omega}^{0}(I)$ are denoted by $\|\cdot\|_{\omega}$ and $(\cdot, \cdot)_{\omega}$, respectively. To account for homogeneous boundary conditions, we define

$$
H_{0, \omega}^{m}(I)=\left\{v \in H_{\omega}^{m}(I): v( \pm 1)=\partial_{x} v( \pm 1)=\cdots=\partial_{x}^{m-1} v( \pm 1)=0\right\}, \quad m=1,2, \ldots,
$$

where $\partial_{x}^{k}=\frac{d^{k}}{d x^{k}}, k \geqslant 1$. The subscript $\omega$ will be omitted from the notations in case of $\omega \equiv 1$.

We denote by $\mathbb{R}$ and $\mathbb{N}$ the sets of all real numbers and non-negative integers, respectively. For any $N \in \mathbb{N}$, let $\mathcal{P}_{N}$ be the set of all algebraic polynomials of degree $\leqslant N$. We denote by $c$ a generic positive constant independent of any function and $N$, and use the expression $A \lesssim B$ to mean that there exists a generic positive constant $c$ such that $A \leqslant c B$.

We recall that the classical Jacobi polynomials $J_{n}^{\alpha, \beta}(x)(n \geqslant 0)$ are defined by

$$
(1-x)^{\alpha}(1+x)^{\beta} J_{n}^{\alpha, \beta}(x)=\frac{(-1)^{n}}{2^{n} n !} \frac{d^{n}}{d x^{n}}\left\{(1-x)^{n+\alpha}(1+x)^{n+\beta}\right\}, \quad x \in I .
$$

Let $\omega^{\alpha, \beta}(x)=(1-x)^{\alpha}(1+x)^{\beta}$ be the Jacobi weight function. For $\alpha, \beta>-1$, the Jacobi polynomials are mutually orthogonal in $L_{\omega^{\alpha, \beta}}^{2}(I)$, i.e.,

$$
\int_{I} J_{n}^{\alpha, \beta}(x) J_{m}^{\alpha, \beta}(x) \omega^{\alpha, \beta}(x) d x=\gamma_{n}^{\alpha, \beta} \delta_{n, m},
$$

where $\delta_{n, m}$ is the Kronecker function, and

$$
\gamma_{n}^{\alpha, \beta}=\frac{2^{\alpha+\beta+1} \Gamma(n+\alpha+1) \Gamma(n+\beta+1)}{(2 n+\alpha+\beta+1) \Gamma(n+1) \Gamma(n+\alpha+\beta+1)} .
$$

The restriction " $\alpha, \beta>-1$ " was imposed to ensure that $\omega^{\alpha, \beta} \in L^{1}(I)$. Some other properties of the Jacobi polynomials to be used in this paper are listed in Appendix A.

In fact, Szegö mentioned in [35] that one can define the Jacobi polynomial with indexes $\alpha$ or $\beta \leqslant-1$, based on the Rodrigues' formula (2.1), which is a polynomial of degree $n$, except for $n+\alpha+\beta+k=0,0 \leqslant k \leqslant l$ (a reduction of the degree in this case). However, the so defined Jacobi polynomials do not satisfy some important properties which hold for $\alpha, \beta>-1$, e.g., they are not mutually orthogonal in $L_{\omega^{\alpha, \beta}}^{2}$ for all $\alpha, \beta$. Hence, they are not quite suitable for numerical computations. We shall define below generalized Jacobi polynomials/functions which inherit selected important properties (of classical Jacobi polynomials) that play essential roles in a spectral approximation.

\subsection{Definition of the $G J P / F s$}

For notational convenience, we introduce the following separable index sets in $\mathbb{R}^{2}$ :

$$
\begin{aligned}
& \aleph_{1}=\{(\alpha, \beta): \alpha, \beta \leqslant-1\}, \quad \aleph_{2}=\{(\alpha, \beta): \alpha \leqslant-1, \beta>-1\}, \\
& \aleph_{3}=\{(\alpha, \beta): \alpha>-1, \beta \leqslant-1\}, \quad \aleph_{4}=\{(\alpha, \beta): \alpha, \beta>-1\} .
\end{aligned}
$$

For any $\alpha, \beta \in \mathbb{R}$, we define

$$
\hat{\alpha}:=\left\{\begin{array}{ll}
-\alpha, & \alpha \leqslant-1, \\
0, & \alpha>-1,
\end{array} \quad \bar{\alpha}:= \begin{cases}-\alpha, & \alpha \leqslant-1, \\
\alpha, & \alpha>-1\end{cases}\right.
$$

(likewise for $\hat{\beta}$ and $\bar{\beta}$ ). Throughout the paper, $\hat{\alpha}, \hat{\beta}$ and $\bar{\alpha}, \bar{\beta}$ are always defined from $\alpha, \beta$ as above.

The symbol $[\alpha]$ represents the largest integer $\leqslant \alpha$, and let

$$
n_{0}:=n_{0}^{\alpha, \beta}:=[\hat{\alpha}]+[\hat{\beta}], \quad n_{1}:=n_{1}^{\alpha, \beta}:=n-n_{0}^{\alpha, \beta} .
$$

The GJP/Fs are defined by

$$
j_{n}^{\alpha, \beta}(x)=\omega^{\hat{\alpha}, \hat{\beta}}(x) J_{n_{1}}^{\bar{\alpha}, \bar{\beta}}(x), \quad \alpha, \beta \in \mathbb{R}, n \geqslant n_{0}^{\alpha, \beta}, x \in I .
$$

We emphasize that $\left\{j_{n}^{\alpha, \beta}\right\}$ are only defined for $n \geqslant n_{0}^{\alpha, \beta}$. This fact is implicitly assumed hereafter. 
We can rewrite (2.6) in a more explicit from:

$$
j_{n}^{\alpha, \beta}(x)= \begin{cases}(1-x)^{-\alpha}(1+x)^{-\beta} J_{n_{1}}^{-\alpha,-\beta}(x), & (\alpha, \beta) \in \aleph_{1}, n_{1}=n-[-\alpha]-[-\beta], \\ (1-x)^{-\alpha} J_{n_{1}}^{-\alpha, \beta}(x), & (\alpha, \beta) \in \aleph_{2}, n_{1}=n-[-\alpha], \\ (1+x)^{-\beta} J_{n_{1}}^{\alpha,-\beta}(x), & (\alpha, \beta) \in \aleph_{3}, n_{1}=n-[-\beta], \\ J_{n}^{\alpha, \beta}(x), & (\alpha, \beta) \in \aleph_{4} .\end{cases}
$$

We see that the GJP/Fs are generated from the classical Jacobi polynomials. In fact, as mentioned in Szegö [35], it is also possible to use the Rodrigues' formula (2.1) to define the Jacobi polynomial $J_{n}^{\alpha, \beta}$ with indexes $\alpha$ or $\beta \leqslant-1$. A particular case is

$$
\left(\begin{array}{l}
n \\
l
\end{array}\right) J_{n}^{-l, \beta}(x)=\left(\begin{array}{c}
n+\beta \\
l
\end{array}\right)\left(\frac{x-1}{2}\right)^{l} J_{n-l}^{l, \beta}(x), \quad 1 \leqslant l \leqslant n .
$$

However, there are very few discussions in [35] about the properties of the so-defined Jacobi polynomials with indexes $\alpha$ or $\beta \leqslant-1$.

\subsection{Basic properties of the GJP/Fs}

The GJP/Fs have the following properties:

- The GJP/F $j_{n}^{\alpha, \beta}(x)$ is a polynomial of degree $n$ if (i) $(\alpha, \beta) \in \aleph_{4}$, or (ii) $\alpha$ and $\beta$ are negative integers. In these cases, it coincides, apart from a constant, with the definition in Szegö [35]. Under the condition (ii), $x=1$ (resp. $x=-1$ ) is the zero of multiplicity of $\hat{\alpha}$ for the polynomial $j_{n}^{\alpha, \beta}(x)$ (resp. $\hat{\beta}$ ). Hence, the GJP/Fs are suitable as base functions to approximate solutions of high-order differential equations with a corresponding set of homogeneous boundary conditions (see Section 3 below).

- We find from (2.2), (2.6) and (2.7) that the GJP/Fs are mutually $L_{\omega^{\alpha, \beta}}^{2}(I)$-orthogonal, i.e.,

$$
\int_{I} j_{n}^{\alpha, \beta}(x) j_{m}^{\alpha, \beta}(x) \omega^{\alpha, \beta}(x) d x=\eta_{n}^{\alpha, \beta} \delta_{m, n}, \quad \text { with } \eta_{n}^{\alpha, \beta}=\gamma_{n_{1}}^{\bar{\alpha}, \bar{\beta}} \text {. }
$$

Here, we used the fact $\bar{\alpha}=2 \hat{\alpha}+\alpha$ and $\bar{\beta}=2 \hat{\beta}+\beta$.

Note that polynomials of the form $(1-x)^{k}(1+x)^{l} J_{n}^{\alpha, \beta}(x)$ (with $\left.\alpha, \beta>-1\right)$ have been frequently used as basis functions to impose boundary conditions, but they do not satisfy the orthogonality relation (2.9). We can also view $\left\{j_{n}^{-k,-l}\right\}$ as the orthogonalization of $\left\{(1-x)^{k}(1+x)^{l} J_{n}^{\alpha, \beta}\right\}$ in $L_{\omega^{-k,-l}}^{2}$.

- They satisfy the Sturm-Liouville equation (see Appendix B.1):

$$
\partial_{x}\left((1-x)^{\alpha+1}(1+x)^{\beta+1} \partial_{x} j_{n}^{\alpha, \beta}(x)\right)+\lambda_{n}^{\alpha, \beta}(1-x)^{\alpha}(1+x)^{\beta} j_{n}^{\alpha, \beta}(x)=0,
$$

where

$$
\lambda_{n}^{\alpha, \beta}= \begin{cases}\left(n_{1}+1\right)\left(n_{1}-\alpha-\beta\right), & (\alpha, \beta) \in \aleph_{1}, \\ n_{1}\left(n_{1}-\alpha+\beta+1\right)-\alpha(\beta+1), & (\alpha, \beta) \in \aleph_{2}, \\ n_{1}\left(n_{1}+\alpha-\beta+1\right)-\beta(\alpha+1), & (\alpha, \beta) \in \aleph_{3}, \\ n_{1}\left(n_{1}+\alpha+\beta+1\right), & (\alpha, \beta) \in \aleph_{4},\end{cases}
$$

and $n_{1}=n-n_{0}^{\alpha, \beta}=n-[\hat{\alpha}]-[\hat{\beta}] \geqslant 0$.

- The definition (2.7) ensures that $\omega^{\alpha+1, \beta+1}(x) j_{n}^{\alpha, \beta}(x) \partial_{x} j_{n}^{\alpha, \beta}(x) \rightarrow 0$ as $|x| \rightarrow 1$. So multiplying $j_{m}^{\alpha, \beta}$ on both sides of (2.10) and integrating by parts, we derive from (2.9) that

$$
\int_{I} \partial_{x} j_{m}^{\alpha, \beta}(x) \partial_{x} j_{n}^{\alpha, \beta}(x) \omega^{\alpha+1, \beta+1}(x) d x=\lambda_{n}^{\alpha, \beta} \eta_{n}^{\alpha, \beta} \delta_{m, n} .
$$

- We infer from (A.3) (see Appendix A) and (2.6) that

$$
j_{n}^{\alpha, \beta}(-x)=(-1)^{n_{1}} j_{n}^{\beta, \alpha}(x), \quad x \in I .
$$


We next study the derivative relations of the GJP/Fs. Let us recall that for $\alpha, \beta>-1$,

$$
\partial_{x} j_{n}^{\alpha, \beta}(x)=\partial_{x} J_{n}^{\alpha, \beta}(x)=\frac{1}{2}(n+\alpha+\beta+1) j_{n-1}^{\alpha+1, \beta+1}(x), \quad n \geqslant 1 .
$$

Unfortunately, the GJP/Fs do not satisfy a similar derivative recurrence relation for all $\alpha, \beta \in \mathbb{R}$. Nevertheless, some useful derivative recurrence relations can be derived.

Lemma 2.1. If one of the following conditions holds
(i) $\alpha, \beta \leqslant-2$;
(ii) $\alpha=-1, \beta \leqslant-2$;
(iii) $\alpha \leqslant-2, \beta=-1$;
(iv) $\alpha=\beta=-1$,

then

$$
\partial_{x} j_{n}^{\alpha, \beta}(x)=-2(n-[-\alpha]-[-\beta]+1) j_{n-1}^{\alpha+1, \beta+1}(x) .
$$

On the other hand, if one of the following conditions holds

$$
\text { (i) } \alpha \leqslant-2, \beta>-1 ; \quad \text { (ii) } \alpha=-1, \beta>-1 \text {, }
$$

then

$$
\partial_{x} j_{n}^{\alpha, \beta}(x)=-(n-[-\alpha]-\alpha) j_{n-1}^{\alpha+1, \beta+1}(x) .
$$

Similarly, if one of the following conditions holds

$$
\text { (i) } \alpha>-1, \beta \leqslant-2 ; \quad \text { (ii) } \alpha>-1, \beta=-1 \text {, }
$$

then

$$
\partial_{x} j_{n}^{\alpha, \beta}(x)=(n-[-\beta]-\beta) j_{n-1}^{\alpha+1, \beta+1}(x) .
$$

The proof of this lemma is given in Appendix B.2.

Applying the formulas in Lemma 2.1 repeatedly, we obtain the following general derivative recurrence relations:

Lemma 2.2. Let $k, l, m \in \mathbb{N}$ and $k, l, m \geqslant 1$. We have

(i) If $\beta>-1$, then

$$
\partial_{x}^{m} j_{n}^{-k, \beta}(x)=D_{m, n, j_{n-m}^{k, \beta}}^{-k+m, \beta+m}(x), \quad n \geqslant \max (k, m),
$$

where

$$
D_{m, n}^{k, \beta}= \begin{cases}(-1)^{m} \prod_{i=0}^{m-1}(n-i), & m \leqslant k, \\ (-1)^{k} \frac{\Gamma(n+m-k+\beta+1)}{2^{m-k} \Gamma(n+\beta+1)} \prod_{i=0}^{k-1}(n-i), & m>k .\end{cases}
$$

(ii) If $\alpha>-1$, then

$$
\partial_{x}^{m} j_{n}^{\alpha,-k}(x)=(-1)^{\mu} D_{m, n, j_{n-m}^{k, \alpha}}^{\alpha+m,-k+m}(x), \quad n \geqslant \max (k, m),
$$

where $\mu=m$ for $m \leqslant k$ and $\mu=k$ for $m>k$.

(iii) If $k \geqslant l$, then

$$
\partial_{x}^{m} j_{n}^{-k,-l}(x)=E_{m, n,}^{k, l} j_{n-m}^{-k+m,-l+m}(x), \quad n \geqslant \max (k+l, m),
$$


where

$$
E_{m, n}^{k, l}=\left\{\begin{array}{l}
(-2)^{m} \prod_{i=1}^{m}(n-l-k+i), \quad m \leqslant l \leqslant k, \\
(-1)^{m} 2^{l}\left(\prod_{i=1}^{l}(n-l-k+i)\right)\left(\prod_{i=0}^{m-l-1}(n-l-i)\right), \quad l<m \leqslant k, \\
(-1)^{k} \frac{\Gamma(n+m-l-k+1)}{2^{m-k-l} \Gamma(n-l+1)}\left(\prod_{i=1}^{l}(n-l-k+i)\right)\left(\prod_{i=0}^{k-l-1}(n-l-i)\right), \quad l<k \leqslant m .
\end{array}\right.
$$

(iv) If $l \geqslant k$, then

$$
\partial_{x}^{m} j_{n}^{-k,-l}(x)=(-1)^{\mu} E_{m, n, j_{n-m}^{l, k}}^{-k+m,-l+m}(x),
$$

where $\mu=0, m, l$ for the cases $m \leqslant k \leqslant l, k<m \leqslant l$ and $k<l \leqslant m$, respectively.

The proof of this lemma is given in Appendix B.3.

\subsection{Approximation properties of the GJP/Fs}

We shall analyze below the approximation properties of generalized Jacobi orthogonal projections, which are useful in the error analysis of spectral-Galerkin methods.

Since $\left\{j_{n}^{\alpha, \beta}\right\}$ forms a complete orthogonal system in $L_{\omega^{\alpha, \beta}}^{2}(I)$, we define

$$
Q_{N}^{\alpha, \beta}:=\operatorname{span}\left\{j_{n_{0}}^{\alpha, \beta}, j_{n_{0}+1}^{\alpha, \beta}, \ldots, j_{N}^{\alpha, \beta}\right\},
$$

and consider the orthogonal projection $\pi_{N}^{\alpha, \beta}: L_{\omega^{\alpha, \beta}}^{2}(I) \rightarrow Q_{N}^{\alpha, \beta}$ defined by

$$
\left(u-\pi_{N}^{\alpha, \beta} u, v_{N}\right)_{\omega^{\alpha, \beta}}=0, \quad \forall v_{N} \in Q_{N}^{\alpha, \beta} .
$$

We shall estimate the projection errors in two different ways. The first approach is based on the Sturm-Liouville equation (2.10). The second one is based on the derivative relations given in Lemma 2.2.

We start with the Sturm-Liouville operator defined by

$$
A_{\alpha, \beta} \phi(x):=-(1-x)^{-\alpha}(1+x)^{-\beta} \partial_{x}\left\{(1-x)^{\alpha+1}(1+x)^{\beta+1} \partial_{x} \phi(x)\right\} .
$$

We recall that $j_{n}^{\alpha, \beta}(x)$ are the eigenfunctions of the Sturm-Liouville operator $A_{\alpha, \beta}$ with the corresponding eigenvalues $\lambda_{n}^{\alpha, \beta}$ (cf. (2.10)), and define the following Sobolev-type spaces associated with the Sturm-Liouville operator:

$$
\begin{aligned}
& \mathcal{D}\left(A_{\alpha, \beta}^{r}\right)=\left\{u: u \in L_{\omega^{\alpha, \beta}}^{2}(I) \text { and } A_{\alpha, \beta}^{q} u \in L_{\omega^{\alpha, \beta}}^{2}(I), 0 \leqslant q \leqslant r\right\}, \quad r \in \mathbb{N}, \\
& \mathcal{D}\left(A_{\alpha, \beta}^{r+1 / 2}\right)=\left\{u: u \in \mathcal{D}\left(A_{\alpha, \beta}^{r}\right) \text { and } \partial_{x} A_{\alpha, \beta}^{r} u \in L_{\omega^{\alpha+1, \beta+1}}^{2}(I)\right\}, \quad r \in \mathbb{N},
\end{aligned}
$$

equipped with the norms

$$
\|u\|_{\mathcal{D}\left(A_{\alpha, \beta}^{r}\right)}=\left\|A_{\alpha, \beta}^{r} u\right\|_{\omega^{\alpha, \beta},}, \quad\|u\|_{\mathcal{D}\left(A_{\alpha, \beta}^{r+1 / 2}\right)}=\left\|\partial_{x} A_{\alpha, \beta}^{r} u\right\|_{\omega^{\alpha+1, \beta+1}} .
$$

Using the identity $A_{\alpha, \beta} j_{n}^{\alpha, \beta}=\lambda_{n}^{\alpha, \beta} j_{n}^{\alpha, \beta}$ repeatedly, we find from (2.9) and (2.12) that for $r \in \mathbb{N}$,

$$
\begin{aligned}
& \|u\|_{\mathcal{D}\left(A_{\alpha, \beta}^{r}\right)}=\left(\sum_{n=0}^{\infty}\left(\lambda_{n}^{\alpha, \beta}\right)^{2 r} \eta_{n}^{\alpha, \beta}\left|\hat{u}_{n}^{\alpha, \beta}\right|^{2}\right)^{1 / 2}, \\
& \|u\|_{\mathcal{D}\left(A_{\alpha, \beta}^{r+1 / 2}\right)}=\left(\sum_{n=0}^{\infty}\left(\lambda_{n}^{\alpha, \beta}\right)^{2 r+1} \eta_{n}^{\alpha, \beta}\left|\hat{u}_{n}^{\alpha, \beta}\right|^{2}\right)^{1 / 2},
\end{aligned}
$$

where $\hat{u}_{n}^{\alpha, \beta}=\left(\eta_{n}^{\alpha, \beta}\right)^{-1}\left(u, j_{n}^{\alpha, \beta}\right)_{\omega^{\alpha, \beta}}$. For real $r>0$, we define the space $\mathcal{D}\left(A_{\alpha, \beta}^{r}\right)$ by space interpolation as in [5]. 
Before we present one of our main results, we make the following observation: For any $v \in \mathcal{D}\left(A_{\alpha, \beta}\right)$, if $\alpha \leqslant-1$ (resp. $\beta \leqslant-1$ ), then $v(x) \rightarrow 0$ as $x \rightarrow 1$ (resp. $x \rightarrow-1$ ), and by the definition of $j_{n}^{\alpha, \beta}$, we have

$$
\omega^{\alpha+1, \beta+1}(x) \partial_{x} j_{n}^{\alpha, \beta}(x)= \begin{cases}(\alpha-\beta+(\alpha+\beta) x) J_{n_{1}}^{-\alpha,-\beta}(x)+\left(1-x^{2}\right) \partial_{x} J_{n_{1}}^{-\alpha,-\beta}(x), & \text { if } \alpha, \beta \leqslant-1 ; \\ (1+x)^{\beta+1}\left\{\alpha J_{n_{1}}^{-\alpha, \beta}(x)+(1-x) \partial_{x} J_{n_{1}}^{-\alpha, \beta}(x)\right\}, & \text { if } \alpha \leqslant-1, \beta>-1 ; \\ (1-x)^{\alpha+1}\left\{-\beta J_{n_{1}}^{\alpha,-\beta}(x)+(1+x) \partial_{x} J_{n_{1}}^{\alpha,-\beta}(x)\right\}, & \text { if } \alpha>-1, \beta \leqslant-1 ; \\ (1-x)^{\alpha+1}(1+x)^{\beta+1} \partial_{x} J_{n}^{\alpha, \beta}(x), & \text { if } \alpha, \beta>-1,\end{cases}
$$

where $n_{1}=n-n_{0} \geqslant 0$. Thus, for any $v \in \mathcal{D}\left(A_{\alpha, \beta}\right)$, we have

$$
\omega^{\alpha+1, \beta+1}(x) v(x) \partial_{x} j_{n}^{\alpha, \beta}(x) \rightarrow 0, \quad \text { as }|x| \rightarrow 1, \forall(\alpha, \beta) \in \mathbb{R}^{2} .
$$

Using the identity $A_{\alpha, \beta} j_{n}^{\alpha, \beta}=\lambda_{n}^{\alpha, \beta} j_{n}^{\alpha, \beta}$ again and integrating by parts, we find that for any $v \in \mathcal{D}\left(A_{\alpha, \beta}\right)$,

$$
\left(v, j_{n}^{\alpha, \beta}\right)_{\omega^{\alpha, \beta}}=\left(\lambda_{n}^{\alpha, \beta}\right)^{-1}\left(v, A_{\alpha, \beta} j_{n}^{\alpha, \beta}\right)_{\omega^{\alpha, \beta}}=\left(\lambda_{n}^{\alpha, \beta}\right)^{-1}\left(A_{\alpha, \beta} v, j_{n}^{\alpha, \beta}\right)_{\omega^{\alpha, \beta}} .
$$

Theorem 2.1. For any $u \in \mathcal{D}\left(A_{\alpha, \beta}^{r / 2}\right), r \in \mathbb{N}$ and $0 \leqslant \mu \leqslant r$,

$$
\left\|\pi_{N}^{\alpha, \beta} u-u\right\|_{\mathcal{D}\left(A_{\alpha, \beta}^{\mu / 2}\right)} \lesssim N^{\mu-r}\|u\|_{\mathcal{D}\left(A_{\alpha, \beta}^{r / 2}\right)}
$$

Proof. The proof follows a similar procedure used for the classical Jacobi projections (cf. [14,9,23]). We first consider even integers, i.e., $r=2 q$ for $q=0,1, \ldots$ We derive from (2.31) that for $\mu \in \mathbb{N}$,

$$
\begin{aligned}
\left\|\pi_{N}^{\alpha, \beta} u-u\right\|_{\mathcal{D}\left(A_{\alpha, \beta}^{\mu / 2}\right)}^{2} & =\sum_{n=N+1}^{\infty}\left(\lambda_{n}^{\alpha, \beta}\right)^{\mu} \eta_{n}^{\alpha, \beta}\left(\hat{u}_{n}^{\alpha, \beta}\right)^{2} \\
& =\sum_{n=N+1}^{\infty}\left(\lambda_{n}^{\alpha, \beta}\right)^{\mu}\left(\eta_{n}^{\alpha, \beta}\right)^{-1}\left(u, j_{n}^{\alpha, \beta}\right)_{\omega^{\alpha, \beta}}^{2} .
\end{aligned}
$$

Using repeatedly the identities $A_{\alpha, \beta} j_{n}^{\alpha, \beta}=\lambda_{n}^{\alpha, \beta} j_{n}^{\alpha, \beta}$ and the relation (2.33), we derive that

$$
\left(u, j_{n}^{\alpha, \beta}\right)_{\omega^{\alpha, \beta}}=\left(\lambda_{n}^{\alpha, \beta}\right)^{-q}\left(A_{\alpha, \beta}^{q} u, j_{n}^{\alpha, \beta}\right)_{\omega^{\alpha, \beta}} .
$$

Hence, by (2.9) and (2.11),

$$
\begin{aligned}
\left\|\pi_{N}^{\alpha, \beta} u-u\right\|_{\mathcal{D}\left(A_{\alpha, \beta}^{\mu}\right)}^{\mu / 2} & =\sum_{n=N+1}^{\infty}\left(\lambda_{n}^{\alpha, \beta}\right)^{\mu}\left(\eta_{n}^{\alpha, \beta}\right)^{-1}\left(u, j_{n}^{\alpha, \beta}\right)_{\omega^{\alpha, \beta}}^{2} \\
& \leqslant \lambda_{N+1}^{\mu-2 q} \sum_{n=N+1}^{\infty}\left(\eta_{n}^{\alpha, \beta}\right)^{-1}\left(A_{\alpha, \beta}^{q} u, j_{n}^{\alpha, \beta}\right)_{\omega^{\alpha, \beta}}^{2} \\
& \lesssim N^{2(\mu-2 q)}\left\|A_{\alpha, \beta}^{q} u\right\|_{\omega^{\alpha, \beta}}^{2} \lesssim N^{2(\mu-r)}\|u\|_{\mathcal{D}\left(A_{\alpha, \beta}^{r / 2}\right)}^{2} .
\end{aligned}
$$

Next, we consider odd integers, i.e., $r=2 q+1$ for $q=0,1, \ldots$ We observe from (2.10), (2.32) and (2.36) that

$$
\left(u, j_{n}^{\alpha, \beta}\right)_{\omega^{\alpha, \beta}}=\left(\lambda_{n}^{\alpha, \beta}\right)^{-q}\left(A_{\alpha, \beta}^{q} u, j_{n}^{\alpha, \beta}\right)_{\omega^{\alpha, \beta}}=\left(\lambda_{n}^{\alpha, \beta}\right)^{-q-1}\left(\partial_{x}\left(A_{\alpha, \beta}^{q} u\right), \partial_{x} j_{n}^{\alpha, \beta}\right)_{\omega^{\alpha+1, \beta+1}} .
$$

Therefore, by (2.11) and (2.12),

$$
\begin{aligned}
\left\|\pi_{N}^{\alpha, \beta} u-u\right\|_{\mathcal{D}\left(A_{\alpha, \beta}^{\mu / 2}\right)}^{2} & =\sum_{n=N+1}^{\infty}\left(\lambda_{n}^{\alpha, \beta}\right)^{\mu}\left(\eta_{n}^{\alpha, \beta}\right)^{-1}\left(u, j_{n}^{\alpha, \beta}\right)_{\omega^{\alpha, \beta}}^{2} \\
& \leqslant \lambda_{N+1}^{\mu-2 q-1} \sum_{n=N+1}^{\infty}\left(\lambda_{n}^{\alpha, \beta}\right)^{-1}\left(\eta_{n}^{\alpha, \beta}\right)^{-1}\left(\partial_{x}\left(A_{\alpha, \beta}^{q} u\right), \partial_{x} j_{n}^{\alpha, \beta}\right)_{\omega^{\alpha+1, \beta+1}}^{2}
\end{aligned}
$$




$$
\begin{aligned}
& \lesssim N^{2(\mu-2 q-1)} \sum_{n=N+1}^{\infty}\left(\lambda_{n}^{\alpha, \beta}\right)^{-1}\left(\eta_{n}^{\alpha, \beta}\right)^{-1}\left(\partial_{x}\left(A_{\alpha, \beta}^{q} u\right), \partial_{x} j_{n}^{\alpha, \beta}\right)_{\omega^{\alpha+1, \beta+1}}^{2} \\
& \lesssim N^{2(\mu-2 q-1)}\left\|\partial_{x}\left(A_{\alpha, \beta}^{q} u\right)\right\|_{\omega^{\alpha+1, \beta+1}}^{2} \lesssim N^{2(\mu-r)}\|u\|_{\mathcal{D}\left(A_{\alpha, \beta}^{r / 2}\right)}^{2} \cdot
\end{aligned}
$$

Finally the desired result with real $\mu$ follows from the previous results and space interpolation.

Theorem 2.1 provides a general approximation result for all $\alpha, \beta \in \mathbb{R}$. However, the norms used in Theorem 2.1 are expressed by the frequencies of $u$ in terms of $j_{n}^{\alpha, \beta}$, whose relation to the derivatives of $u$ is not straightforward. Next, we derive some approximation results which are expressed in terms of derivatives of $u$.

We introduce the space

$$
B_{\omega^{\alpha, \beta}}^{r}(I):=\left\{u: u \text { is measurable on } I \text { and }\|u\|_{r, \omega^{\alpha, \beta}}<\infty\right\}, \quad r \in \mathbb{N},
$$

equipped with the norm and semi-norm

$$
\|u\|_{B_{\omega^{\alpha}, \beta}^{r}}=\left(\sum_{k=0}^{r}\left\|\partial_{x}^{k} u\right\|_{\omega^{\alpha+k, \beta+k}}^{2}\right)^{1 / 2}, \quad|u|_{B_{\omega^{\alpha, \beta}}^{r}}=\left\|\partial_{x}^{r} u\right\|_{\omega^{\alpha+r, \beta+r}} .
$$

For real $r>0$, we define the space $H_{\omega^{\alpha, \beta}}^{r}(I)$ by space interpolation as in [5].

Theorem 2.2. Let $k, l \geqslant 1$ and $k, l \in \mathbb{N}$. If one of the following conditions holds:

$$
\text { (i) } \alpha=-k, \beta>-1 ; \quad \text { (ii) } \alpha>-1, \beta=-l ; \quad \text { (iii) } \alpha=-k, \beta=-l \text {, }
$$

then for any $u \in B_{\omega^{\alpha, \beta}}^{r}(I), r \in \mathbb{N}, r \geqslant 1$ and $0 \leqslant \mu \leqslant r$,

$$
\left\|\pi_{N}^{\alpha, \beta} u-u\right\|_{B_{\omega^{\alpha, \beta}}^{\mu}} \lesssim N^{\mu-r}\left\|\partial_{x}^{r} u\right\|_{\omega^{\alpha+r, \beta+r}} .
$$

Proof. We first prove (2.39) with (i). In this case,

$$
\pi_{N}^{-k, \beta} u(x)-u(x)=-\sum_{n=N+1}^{\infty} \hat{u}_{n} j_{n}^{-k, \beta}(x), \quad \text { with } \hat{u}_{n}=\frac{\left(u, j_{n}^{-k, \beta}\right)_{\omega^{-k, \beta}}}{\left\|j_{n}^{-k, \beta}\right\|_{\omega^{-k, \beta}}^{2}} .
$$

As a consequence of (2.9) and (2.21)-(2.22), we have the orthogonality:

$$
\int_{I} \partial_{x}^{m} j_{n}^{-k, \beta}(x) \partial_{x}^{m} j_{n^{\prime}}^{-k, \beta}(x) \omega^{-k+m, \beta+m}(x) d x=\left(D_{m, n}^{k, \beta}\right)^{2} \eta_{n-m}^{-k+m, \beta+m} \delta_{n, n^{\prime}} .
$$

Thanks to (2.41), we deduce from (2.40) that for $\mu \in \mathbb{N}$,

$$
\begin{aligned}
\left\|\partial_{x}^{\mu}\left(\pi_{N}^{-k, \beta} u-u\right)\right\|_{\omega^{-k+\mu, \beta+\mu}}^{2} & =\sum_{n=N+1}^{\infty}\left(D_{\mu, n}^{k, \beta}\right)^{2} \hat{u}_{n}^{2} \eta_{n-\mu}^{-k+\mu, \beta+\mu} \\
& \leqslant C_{N, \mu, r}^{k, \beta} \sum_{n=N+1}^{\infty}\left(D_{r, n}^{k, \beta}\right)^{2} \hat{u}_{n}^{2} \eta_{n-r}^{-k+r, \beta+r} \leqslant C_{N, \mu, r}^{k, \beta}\left\|\partial_{x}^{r} u\right\|_{\omega^{-k+r, \beta+r}}^{2},
\end{aligned}
$$

where

$$
C_{N, \mu, r}^{k, \beta}=\max _{n>N}\left\{\frac{\left(D_{\mu, n}^{k, \beta}\right)^{2} \eta_{n-\mu}^{-k+\mu, \beta+\mu}}{\left(D_{r, n}^{k, \beta}\right)^{2} \eta_{n-r}^{-k+r, \beta+r}}\right\} .
$$

We now estimate the upper bound of $C_{N, \mu, r}^{k, \beta}$. By using the Stirling formula (cf. [15]),

$$
\Gamma(s+1)=\sqrt{2 \pi s} s s^{s} e^{-s}\left(1+\mathrm{O}\left(s^{-1 / 5}\right)\right), \quad s \gg 1,
$$


we derive from (2.3), (2.9) and (2.22) that for given $\bar{\alpha}, \bar{\beta}, k, \mu, \gamma$,

$$
\gamma_{n}^{\bar{\alpha}, \bar{\beta}} \sim n^{-1}, \quad \eta_{n-\mu}^{-k+\mu, \beta+\mu} \sim \eta_{n-r}^{-k+r, \beta+r} \sim n^{-1}, \quad \frac{D_{\mu, n}^{k, \beta}}{D_{r, n}^{k, \beta}} \sim N^{\mu-r}, \quad n \gg 1 .
$$

The above facts lead to $C_{N, \mu, r}^{\alpha, \beta} \lesssim N^{2 \mu-2 r}$. This completes the proof of (2.39) with (i).

The other two cases can be proved similarly.

Remark 2.1. The results for the classical Jacobi polynomials with $\alpha, \beta>-1$ were proved in [26]. The same results for $\mu=0$ or $\alpha=\beta$ were also given in [19,3], respectively. The results for the case $\alpha=-k$ and $\beta=-l$ were announced (without proof) in [25].

\section{Applications}

An important application of GJP/Fs is that they form natural basis functions for spectral-Galerkin approximations of differential equations. For example, one can verify (see Appendix B) that

$$
\begin{aligned}
& j_{n}^{-1,-1}(x)=\frac{2(n-1)}{2 n-1}\left(L_{n-2}(x)-L_{n}(x)\right), \\
& j_{n}^{-2,-1}(x)=\frac{2(n-2)}{2 n-3}\left(L_{n-3}(x)-\frac{2 n-3}{2 n-1} L_{n-2}(x)-L_{n-1}(x)+\frac{2 n-3}{2 n-1} L_{n}(x)\right), \\
& j_{n}^{-1,-2}(x)=\frac{2(n-2)}{2 n-3}\left(L_{n-3}(x)+\frac{2 n-3}{2 n-1} L_{n-2}(x)-L_{n-1}(x)-\frac{2 n-3}{2 n-1} L_{n}(x)\right), \\
& j_{n}^{-2,-2}(x)=\frac{4(n-2)(n-3)}{(2 n-3)(2 n-5)}\left(L_{n-4}(x)-\frac{2(2 n-3)}{2 n-1} L_{n-2}(x)+\frac{2 n-5}{2 n-1} L_{n}(x)\right),
\end{aligned}
$$

where $L_{k}(x)$ is the Legendre polynomial of $k$-th degree. The GJP/Fs in (3.1) and (3.4) were used in [32] as basis functions to approximate the solutions of second- and forth-order equations with homogeneous Dirichlet boundary conditions, while the GJP/Fs in (3.2) and (3.3) were used as basis functions for the test and trial spaces in the dualPetrov-Galerkin method for third-order differential equations in [33].

A main advantage of using the GJP/Fs as basis functions is that the GJP/Fs satisfy all given boundary conditions of the underlying problem. Hence, there is no need to construct special quadratures involving derivatives at end-points as in a collocation approach [7,27,29]. for third-order equations and in [7] for fourth-order equations. The spectral approximations using GJP/Fs lead to well-conditioned, sparse for problems with constant or polynomial coefficients (cf. [32,33]), systems that can be efficiently implemented. Moreover, using the GJP/Fs simplifies theoretical analysis, and leads to more precise error estimates as demonstrated below.

\subsection{Spectral-Galerkin methods for high order equations}

We consider the following $2 m$-th order linear equation:

$$
\begin{aligned}
& \mathcal{L}_{2 m} u:=(-1)^{m} b_{0} u^{(2 m)}+\sum_{k=0}^{2 m-1} b_{2 m-k} u^{(k)}=f, \quad \text { in } I, m \geqslant 1, \\
& u^{(k)}( \pm 1)=0, \quad 0 \leqslant k \leqslant m-1,
\end{aligned}
$$

where $\left\{b_{j}\right\}_{0 \leqslant j \leqslant 2 m}$ and $f$ are given, and we assume $b_{0}>0$. We introduce the bilinear form associated with (3.5):

$$
\begin{aligned}
a_{m}(u, v)= & \left(b_{0} \partial_{x}^{m} u, \partial_{x}^{m} v\right)+(-1)^{m}\left(b_{1} \partial_{x}^{m-1} u, \partial_{x}^{m} v\right) \\
& +(-1)^{m-1}\left(b_{2} \partial_{x}^{m-1} u, \partial_{x}^{m-1} v\right)+\cdots+\left(b_{2 m} u, v\right), \quad \forall u, v \in H^{m}(I) .
\end{aligned}
$$

As usual, we assume that the bilinear form is continuous and elliptic in $H_{0}^{m}(I)$, i.e., 


$$
\begin{aligned}
& \left|a_{m}(u, v)\right| \leqslant C_{0}|u|_{m}|v|_{m}, \quad \forall u, v \in H_{0}^{m}(I), \\
& a_{m}(u, u) \geqslant C_{1}|u|_{m}^{2}, \quad \forall u \in H_{0}^{m}(I)
\end{aligned}
$$

where $C_{0}$ and $C_{1}$ are two positive constants depending only on $b_{j}, 0 \leqslant j \leqslant 2 m$.

The variational formulation for (3.5) is: Given $f \in H^{-m}(I)$, find $u \in H_{0}^{m}(I)$ such that

$$
a_{m}(u, v)=(f, v), \quad \forall v \in H_{0}^{m}(I),
$$

and the corresponding spectral-Galerkin approximation is: Given $f \in C(\bar{I})$, find $u_{N} \in V_{N}:=\mathcal{P}_{N} \cap H_{0}^{m}(I)$ such that

$$
a_{m}\left(u_{N}, v_{N}\right)=\left(f, v_{N}\right)_{N}, \quad \forall v_{N} \in V_{N},
$$

where $(\cdot, \cdot)_{N}$ is the inner product associated to the Legendre-Gauss-Lobatto quadrature. The well-posedness of (3.8) and (3.9) is ensured by (3.7a)-(3.7b).

\subsection{Error estimates}

Let us denote $\pi_{N}^{m}=\pi_{N}^{-m,-m}$. We note immediately that

$$
\begin{aligned}
\left(\partial_{x}^{m}\left(\pi_{N}^{m} u-u\right), \partial_{x}^{m} v_{N}\right) & =(-1)^{m}\left(\pi_{N}^{m} u-u, \partial_{x}^{2 m} v_{N}\right) \\
& =(-1)^{m}\left(\pi_{N}^{m} u-u, \omega^{m, m} \partial_{x}^{2 m} v_{N}\right)_{\omega^{-m,-m}}=0, \quad \forall v_{N} \in V_{N},
\end{aligned}
$$

which is a consequence of (2.28) and the fact $\omega^{m, m} \partial_{x}^{2 m} v_{N} \in V_{N}$. In other words, $\pi_{N}^{m}$ is simultaneously orthogonal projectors associated with $(\cdot, \cdot)_{\omega^{-m,-m}}$ and $\left(\partial_{x}^{m} \cdot, \partial_{x}^{m} \cdot\right)$.

For simplicity, we assume that $\left\{b_{j}\right\}$ are constants, and let $u$ and $u_{N}$ be respectively the solutions of (3.8) and (3.9). Then, we have the following result:

Theorem 3.1. Assuming $u \in H_{0}^{m}(I) \cap B_{\omega^{-m,-m}}^{r}(I)$ and $f\left(1-x^{2}\right)^{m} \in B_{\omega^{0,0}}^{\rho}(I), m, r, \rho \in \mathbb{N}$ with $1 \leqslant m \leqslant r, 1 \leqslant \rho$, then for $0 \leqslant \mu \leqslant m$, we have

$$
\left\|u-u_{N}\right\|_{\mu} \lesssim N^{\mu-r}\left\|\partial_{x}^{r} u\right\|_{\omega^{r-m, r-m}}+N^{-\rho}\left\|\partial_{x}^{\rho}\left(f\left(1-x^{2}\right)^{m}\right)\right\|_{\omega^{\rho, \rho}} .
$$

Proof. We denote $\hat{e}_{N}=\pi_{N}^{m} u-u_{N}$ and $e_{N}=u-u_{N}=\left(u-\pi_{N}^{m} u\right)+\hat{e}_{N}$.

We first prove (3.11) for $\mu=m$. We derive from (3.8) and (3.9) that

$$
a_{m}\left(\hat{e}_{N}, v_{N}\right)=a_{m}\left(\pi_{N}^{m} u-u, v_{N}\right)+\left(f, v_{N}\right)-\left(f, v_{N}\right)_{N}, \quad v_{N} \in V_{N} .
$$

By using the Hardy inequality (cf., for example, Section A.14 in [13]), it is easy to show that

$$
\int_{I} v^{2}\left(1-x^{2}\right)^{-2 m} d x \lesssim \int_{I}\left(\partial_{x} v\right)^{2}\left(1-x^{2}\right)^{-2 m+2} d x \lesssim \cdots \lesssim \int_{I}\left(\partial_{x}^{m} v\right)^{2} d x, \quad \forall v \in H_{0}^{m}(I) .
$$

For $v_{N} \in V_{N}$, let $\tilde{f}=f\left(1-x^{2}\right)^{m}$ and $\tilde{v}_{N}=v_{N}\left(1-x^{2}\right)^{-m}$, then by using the properties of the Legendre-GaussLobatto quadrature (cf. [13]) and (3.13),

$$
\begin{aligned}
\left(f, v_{N}\right)-\left(f, v_{N}\right)_{N} & =\left(\tilde{f}, \tilde{v}_{N}\right)-\left(\tilde{f}, \tilde{v}_{N}\right)_{N}=\left(\tilde{f}-\pi_{N-1}^{0} \tilde{f}, \tilde{v}_{N}\right)-\left(I_{N} \tilde{f}-\pi_{N-1}^{0} \tilde{f}, \tilde{v}_{N}\right)_{N} \\
& \lesssim\left(\left\|\tilde{f}-\pi_{N-1}^{0} \tilde{f}\right\|+\left\|I_{N} \tilde{f}-\pi_{N-1}^{0} \tilde{f}\right\|\right)\left\|\tilde{v}_{N}\right\| \\
& \lesssim\left(\left\|\tilde{f}-\pi_{N-1}^{0} \tilde{f}\right\|+\left\|\tilde{f}-I_{N} \tilde{f}\right\|\right)\left\|\partial_{x}^{m} v_{N}\right\| \\
& \leqslant C\left(\left\|\tilde{f}-\pi_{N-1}^{0} \tilde{f}\right\|^{2}+\left\|\tilde{f}-I_{N} \tilde{f}\right\|^{2}\right)+\frac{C_{1}}{2}\left\|v_{N}\right\|_{m}^{2} .
\end{aligned}
$$

We recall from Theorem 4.10 in [26] and Theorem 2.2 with $(\alpha, \beta)=(0,0)$ that

$$
\left\|\tilde{f}-\pi_{N-1}^{0} \tilde{f}\right\|+\left\|\tilde{f}-I_{N} \tilde{f}\right\| \lesssim N^{-\rho}\left\|\partial_{x}^{\rho} \tilde{f}\right\|_{\omega^{\rho, \rho}} .
$$

Thanks to (3.10), the first term involving the derivative of the highest order $m$ vanishes in the expression of $a_{m}\left(\pi_{N}^{m} u-u, v_{N}\right)$. Moreover, we have from (2.39) with condition (iii) that for certain suitable small $\varepsilon>0,0 \leqslant$ $k \leqslant m-1, k \leqslant l \leqslant k+1, k+l \leqslant 2 m-1$ and $k, l \in \mathbb{N}$, 


$$
\begin{aligned}
\left|\left(\partial_{x}^{k}\left(\pi_{N}^{m} u-u\right), \partial_{x}^{l} v_{N}\right)\right| & \leqslant\left\|\partial_{x}^{k}\left(\pi_{N}^{m} u-u\right)\right\|_{\omega^{-m+k,-m+k}}\left\|\partial_{x}^{l} v_{N}\right\|_{\omega^{m-k, m-k}} \\
& \lesssim N^{k-r}\left\|\partial_{x}^{r} u\right\|_{\omega^{r-m, r-m}}\left\|v_{N}\right\|_{l} \\
& \leqslant \varepsilon\left\|v_{N}\right\|_{l}^{2}+\frac{c}{4 \varepsilon} N^{2 k-2 r}\left\|\partial_{x}^{r} u\right\|_{\omega^{r-m, r-m}}^{2} .
\end{aligned}
$$

Taking $v_{N}=\hat{e}_{N}$ in (3.12) and (3.16), we derive from (3.6), (3.7b), (3.14) and 3.15 that

$$
\begin{aligned}
\frac{C_{1}}{2}\left\|\hat{e}_{N}\right\|_{m}^{2} & \lesssim \varepsilon\left\|\hat{e}_{N}\right\|_{m}^{2}+\left(\sum_{k=0}^{m-1} N^{2 k-2 r}\right)\left\|\partial_{x}^{r} u\right\|_{\omega^{r-m, r-m}}^{2}+N^{-2 \rho}\left\|\partial_{x}^{\rho} \tilde{f}\right\|_{\omega^{\rho, \rho}}^{2} \\
& \lesssim \varepsilon\left\|\hat{e}_{N}\right\|_{m}^{2}+N^{2 m-2 r-2}\left\|\partial_{x}^{r} u\right\|_{\omega^{r-m, r-m}}^{2}+N^{-2 \rho}\left\|\partial_{x}^{\rho} \tilde{f}\right\|_{\omega^{\rho, \rho}}^{2} .
\end{aligned}
$$

Thus,

$$
\left\|\hat{e}_{N}\right\|_{m} \lesssim N^{m-r-1}\left\|\partial_{x}^{r} u\right\|_{\omega^{r-m, r-m}}+N^{-\rho}\left\|\partial_{x}^{\rho} \tilde{f}\right\|_{\omega^{\rho, \rho}} .
$$

On the other hand, we have from (2.39) with condition (iii) that

$$
\left\|\pi_{N}^{m} u-u\right\|_{m} \lesssim\left\|\pi_{N}^{m} u-u\right\|_{B_{\omega^{-m},-m}^{m}} \lesssim N^{m-r}\left\|\partial_{x}^{r} u\right\|_{\omega^{r-m, r-m}} .
$$

So (3.11) follows from the triangle inequality, (3.18) and the above estimate.

We now turn to the case $\mu=0$. For given $g \in L^{2}(I)$, we consider the auxiliary problem: Find $w \in H_{0}^{m}(I)$ such that

$$
a_{m}(z, w)=(g, z), \quad \forall z \in H_{0}^{m}(I) .
$$

We know from (3.7a) and (3.7b) that (3.19) has a unique solution with the regularity

$$
\|w\|_{2 m} \lesssim\|g\| \text {. }
$$

From (3.8) and (3.9),

$$
a_{m}\left(u-u_{N}, v_{N}\right)=\left(f, v_{N}\right)-\left(f, v_{N}\right)_{N}, \quad \forall v_{N} \in V_{N} .
$$

Taking $z=u-u_{N}$ in (3.19), we find from Theorem 2.2, (3.7a), (3.11), (3.20)-(3.21) and (3.14) that

$$
\begin{aligned}
\left(u-u_{N}, g\right) & =a_{m}\left(u-u_{N}, w\right)=a_{m}\left(u-u_{N}, w-\pi_{N}^{m} w\right)+\left(f, \pi_{N}^{m} w\right)-\left(f, \pi_{N}^{m} w\right)_{N} \\
& \lesssim\left\|u-u_{N}\right\|_{m}\left\|\pi_{N}^{m} w-w\right\|_{m}+\left(\left\|\tilde{f}-\pi_{N-1}^{0} \tilde{f}\right\|+\left\|I_{N} \tilde{f}-\pi_{N-1}^{0} \tilde{f}\right\|\right)\left\|\partial_{x}^{m} \pi_{N}^{m} w\right\| \\
& \lesssim N^{-r}\left\|\partial_{x}^{r} u\right\|_{\omega^{r-m, r-m}}\left\|\partial_{x}^{2 m} w\right\|_{\omega^{m, m}}+N^{-\rho}\left\|\partial_{x}^{\rho} \tilde{f}\right\|_{\omega^{\rho, \rho}}\|w\|_{m} \\
& \lesssim\left(N^{-r}\left\|\partial_{x}^{r} u\right\|_{\omega^{r-m, r-m}}+N^{-\rho}\left\|\partial_{x}^{\rho} \tilde{f}\right\|_{\omega^{\rho, \rho}}\right)\|g\| .
\end{aligned}
$$

Consequently,

$$
\left\|u-u_{N}\right\|=\sup _{\substack{g \in L^{2}(I) \\ g \neq 0}} \frac{\left|\left(u-u_{N}, g\right)\right|}{\|g\|} \lesssim N^{-r}\|u\|_{\omega^{r-m, r-m}}+N^{-\rho}\left\|\partial_{x}^{\rho} \tilde{f}\right\|_{\omega^{\rho, \rho}} .
$$

This implies the result with $\mu=0$.

For $0<\mu<m$, let $\theta=1-\frac{\mu}{m}$. Clearly $0<\theta<1$. Since $H^{m}(I)$ is continuously embedded and dense in $L^{2}(I)$, we can define the interpolation space $\left[H^{m}(I), L^{2}(I)\right]_{\theta}$ as in [5]. Indeed, as is shown in Theorem 1.6 of [9] (see also [22]), $\left[H^{m}(I), L^{2}(I)\right]_{\theta}=H^{(1-\theta) m}(I)=H^{\mu}(I)$. Therefore, by the Gagliardo-Nirenberg inequality and the previous results,

$$
\left\|u-u_{N}\right\|_{\mu} \leqslant\left\|u-u_{N}\right\|_{m}^{1-\theta}\left\|u-u_{N}\right\|^{\theta} \lesssim N^{\mu-r}|u|_{r, \omega^{-m,-m}}+N^{-\rho}\left\|\partial_{x}^{\rho} \tilde{f}\right\|_{\omega^{\rho, \rho}} .
$$

This ends the proof.

Remark 3.1. Using the GJP/F approximation not only greatly simplifies the error analysis, but also leads to more precise error estimates. For instance, if we use the $H_{0}^{m}$-orthogonal projection results in [8] and [22], then the best error estimate will be

$$
\left\|u-u_{N}\right\|_{m} \lesssim N^{m-r}\|u\|_{r}+N^{-\rho}\|f\|_{\rho}, \quad 0 \leqslant m \leqslant r .
$$


Therefore, the result (3.11) is much sharper than (3.22) for problems with singularities at the endpoints. As an example, let

$$
u(x)=(1-x)^{\gamma} v(x), \quad v \in C^{\infty}(I), \gamma>m, x \in I,
$$

be a solution of (3.5). It can be easily checked that $u \in H^{\gamma+1 / 2-\varepsilon}(I) \cap B_{\omega^{-m,-m}}^{2 \gamma-m+1-\varepsilon}(I)(\forall \varepsilon>0)$ and $f \in$ $H^{\gamma-2 m+1 / 2-\varepsilon}(I), f\left(1-x^{2}\right)^{m} \in B_{\omega^{0,0}}^{2(\gamma-m)+1-\varepsilon}(I)(\forall \varepsilon>0)$. Hence, Theorem 3.1 with $\mu=m$ implies that

$$
\left\|u-u_{N}\right\|_{m} \lesssim N^{-2 \gamma+2 m-1+\varepsilon},
$$

while the usual analysis (cf. Bernardi and Maday [7]) only leads to

$$
\left\|u-u_{N}\right\|_{3} \lesssim N^{-\gamma+2 m+1 / 2+\varepsilon} .
$$

\subsection{Matrix form of (3.9)}

In view of the homogeneous boundary conditions satisfied by $j_{k}^{-m,-m}$, we have

$$
V_{N}=\operatorname{span}\left\{j_{2 m}^{-m,-m}, j_{2 m+1}^{-m,-m}, \ldots, j_{N}^{-m,-m}\right\} .
$$

Using the facts that $\omega^{m, m} \partial_{x}^{2 m} j_{l}^{-m,-m} \in V_{l}$ and $j_{k}^{-m,-m}$ is orthogonal to $V_{l}$ if $k>l$, we find that

$$
\begin{aligned}
\left(\partial_{x}^{m} j_{k}^{-m,-m}, \partial_{x}^{m} j_{l}^{-m,-m}\right) & =(-1)^{m}\left(j_{k}^{-m,-m}, \partial_{x}^{2 m} j_{l}^{-m,-m}\right) \\
& =\left(j_{k}^{-m,-m}, \omega^{m, m} \partial_{x}^{2 m} j_{l}^{-m,-m}\right)_{\omega^{-m,-m}}=0 .
\end{aligned}
$$

By symmetry, the same is true if $k<l$. Hence, letting $\phi_{k}(x)=c_{m, k} j_{k}^{-m,-m}$ with a suitable $c_{m, k}$, we can have

$$
\left(\partial_{x}^{m} \phi_{k}, \partial_{x}^{m} \phi_{l}\right)=\delta_{k l} .
$$

Hence, by setting

$$
\begin{aligned}
& f_{k}=\left(f, \phi_{k}\right), \quad \mathbf{f}=\left(f_{2 m}, f_{2 m+1}, \ldots, f_{N}\right)^{T}, \\
& u_{N}=\sum_{l=2 m}^{N} \hat{u}_{l} \phi_{l}, \quad \mathbf{u}=\left(\hat{u}_{2 m}, \hat{u}_{2 m+1}, \ldots, \hat{u}_{N}\right)^{T}, \\
& a_{k l}=a_{m}\left(\phi_{l}, \phi_{k}\right), \quad A=\left(a_{k l}\right)_{2 m \leqslant k, l \leqslant N},
\end{aligned}
$$

the matrix system associated with (3.9) becomes

$$
A \mathbf{u}=\mathbf{f} .
$$

Thanks to (3.7a)-(3.7b), we have

$$
C_{0}\|\mathbf{u}\|_{l^{2}}^{2}=C_{0}\left|u_{N}\right|_{m}^{2} \leqslant a_{m}\left(u_{N}, u_{N}\right)=(A \mathbf{u}, \mathbf{u})_{l^{2}} \leqslant C_{1}\left|u_{N}\right|_{m}^{2}=C_{1}\|\mathbf{u}\|_{l^{2}}^{2},
$$

which implies that cond $(A) \leqslant C_{1} / C_{0}$ and is independent of $N$. It can be easily shown that $A$ is a sparse matrix with bandwidth $2 m+1$. The same argument as above shows that (3.28) is still valid for problems with variable coefficients as long as (3.7a)-(3.7b) are satisfied. Therefore, even though $A$ becomes full for problems with variable coefficients but the product of $A$ with a vector $\bar{x}$ can be computed efficiently without the explicit knowledge of the entries of $A$ so the associated linear system can still be solved efficiently with a suitable iterative method such as the Conjugate Gradient method.

The generalized Jacobi polynomials/functions were also successfully used for numerical solutions of partial differential equations of odd orders (cf. [25,34]). 


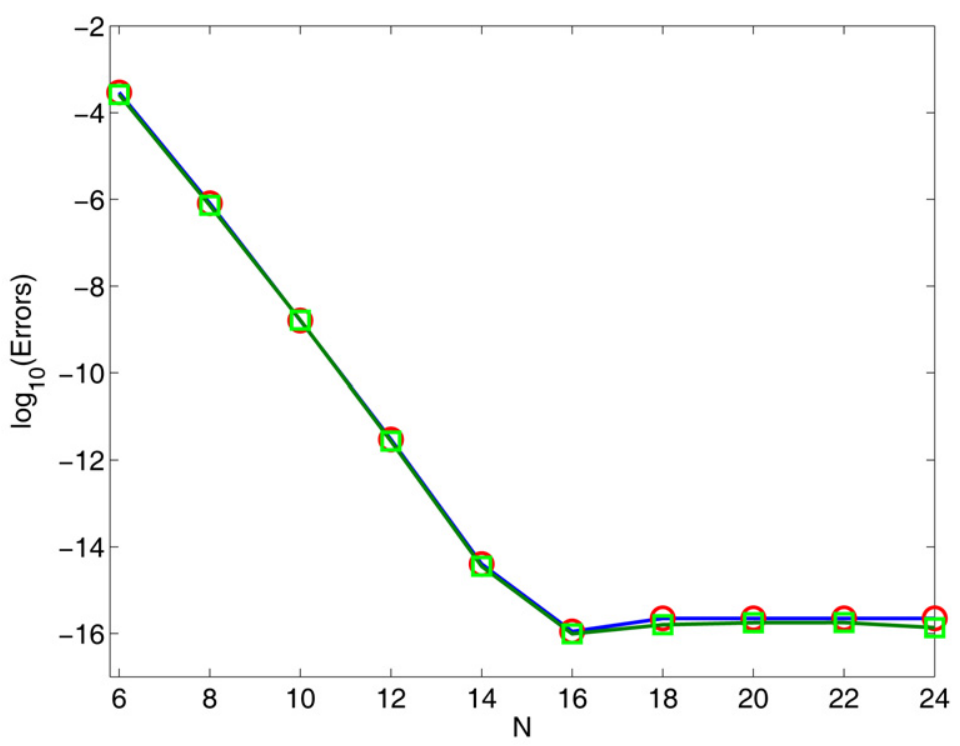

Fig. 1. The maximum pointwise error (marker “o") and the $L^{2}$-error (marker " $\square$ ") against various $N$ in semi-log scale for Example 1.

\section{Numerical results}

We present some numerical examples to illustrate the performance of the proposed spectral methods using generalized Jacobi polynomials as basis functions. As an example, we consider the sixth-order equations, which are known to arise in astrophysics [1,11]. In the computations, we use the spectral-Galerkin scheme (3.9) with $m=3$ and the generalized Jacobi polynomials $\left\{J_{m}^{-3,-3}\right\}$ as the basis functions.

We first consider an example discussed in [17], where the numerical solutions are obtained by a Sinc-Galerkin method.

\section{Example 1. Consider}

$$
u^{(6)}(x)-u(x)=f(x), \quad x \in(-1,1),
$$

with boundary conditions for $u( \pm 1), u^{\prime}( \pm 1), u^{\prime \prime}( \pm 1)$ and $f(x)$ such that the exact solution is $u(x)=(1-x) e^{x}$.

In Fig. 1, we plot the maximum pointwise error and the $L^{2}$-error against various $N$. It is clear that the errors decay exponentially fast, consistent with the results in Theorem 3.1 since both the solution $u$ and the function $f$ are analytic. Note that with the same computational cost, say, $N=16$, the Sinc-method in [17] only achieves an accuracy $\mathrm{O}\left(10^{-4}\right)$, see Table 4.3 in [17], while our method is much more accurate.

Example 2. We consider (4.1) with boundary conditions for $u( \pm 1), u^{\prime}( \pm 1), u^{\prime \prime}( \pm 1)$ and $f(x)$ such that the exact solution is

$$
u(x)=(1+x)^{\gamma} e^{x}, \quad x \in(-1,1) .
$$

When $\gamma$ is not an integer, the solution has a finite regularity and it can be easily checked that (cf. Remark 3.1) $u \in B_{\omega^{-3,-3}}^{2 \gamma-2-\varepsilon}(I), f\left(1-x^{2}\right)^{3} \in B_{\omega^{0,0}}^{2 \gamma-5-\varepsilon}(I)(\forall \varepsilon>0)$. Hence, Theorem 3.1 with $m=3$ and $\mu=3$ implies that

$$
\left\|u-u_{N}\right\|_{3} \lesssim N^{\varepsilon-2 \gamma+5} \quad(\forall \varepsilon>0) .
$$

We plot in Fig. 2 the $H^{3}$-error against various $N$ with $\gamma=3.1,3.5,3.8,4.2$. Note that for these values of $\gamma, f$ is not even in $L^{2}(I)$. The "approximate" slopes of these lines are respectively $-1.19,-2.01,-2.65$ and -3.49 . These convergence rates are very close to the predicted convergence rate of $2 \gamma-5$ in (4.2). 


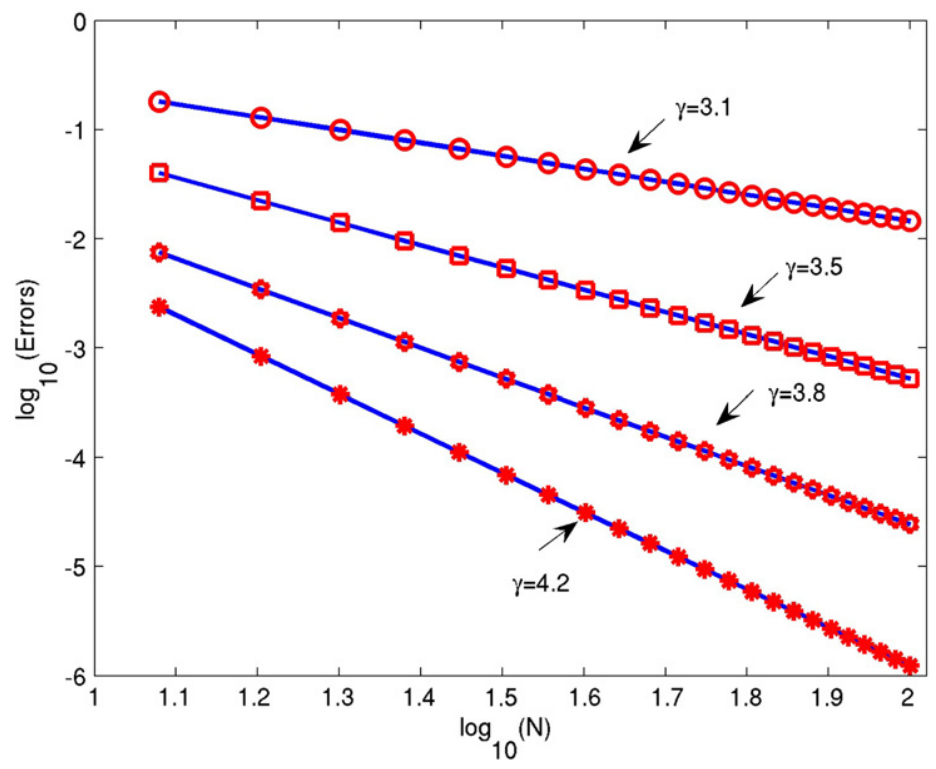

Fig. 2. $H^{3}$-errors against various $N$ in $\log -\log$ scale for Example 2 with several $\gamma$.

\section{Concluding remarks}

We introduced in this paper a family of generalized Jacobi polynomials/functions with indexes $\alpha, \beta \in \mathbb{R}$ based on the principle that they are mutually orthogonal with respect to the corresponding Jacobi weights and that they inherit selected important properties of the classical Jacobi polynomials. We established two sets of approximation results by using the Sturm-Liouville operator and the derivative recurrence relations.

An important application of GJP/Fs is to serve as basis functions for spectral approximations of differential equations with suitable boundary conditions which are automatically satisfied by corresponding GJP/Fs. This is especially convenient for high-order differential equations. Unlike in a collocation method for which special quadratures involving derivatives at the end points need to be developed, the implementations using GJP/Fs are simple and straightforward. Moreover, the use of generalized Jacobi polynomials/functions leads to much simplified analysis, more precise error estimates and well conditioned algorithms.

\section{Appendix A. Properties of the classical Jacobi polynomials}

The classical Jacobi polynomials are the eigenfunctions of the Sturm-Liouville problem:

$$
\partial_{x}\left((1-x)^{\alpha+1}(1+x)^{\beta+1} \partial_{x} J_{n}^{\alpha, \beta}(x)\right)+\mu_{n}^{\alpha, \beta}(1-x)^{\alpha}(1+x)^{\beta} J_{n}^{\alpha, \beta}(x)=0, \quad n \geqslant 0,
$$

with the corresponding eigenvalues $\mu_{n}^{\alpha, \beta}=n(n+\alpha+\beta+1)$. An alternative form of (A.1) is (see [35])

$$
\left(1-x^{2}\right) \partial_{x}^{2} Y_{n}+[\alpha-\beta+(\alpha+\beta-2) x] \partial_{x} Y_{n}+(n+1)(n+\alpha+\beta) Y_{n}=0
$$

where $Y_{n}(x)=\omega^{\alpha, \beta}(x) J_{n}^{\alpha, \beta}(x)$ and $\omega^{\alpha, \beta}(x)=(1-x)^{\alpha}(1+x)^{\beta}$.

The classical Jacobi polynomials with indexes $\alpha, \beta>-1$ satisfy the following recurrence relations (see Szegö [35], Askey [2] and Rainville [31]):

$$
\begin{aligned}
& J_{n}^{\alpha, \beta}(-x)=(-1)^{n} J_{n}^{\beta, \alpha}(x) \\
& J_{n-1}^{\alpha, \beta}(x)=J_{n}^{\alpha, \beta-1}(x)-J_{n}^{\alpha-1, \beta}(x), \quad \alpha, \beta>0, n \geqslant 1 ; \\
& J_{n}^{\alpha, \beta}(x)=\frac{1}{n+\alpha+\beta}\left[(n+\beta) J_{n}^{\alpha, \beta-1}(x)+(n+\alpha) J_{n}^{\alpha-1, \beta}(x)\right], \quad \alpha, \beta>0 ; \\
& (1-x) J_{n}^{\alpha+1, \beta}(x)=\frac{2}{2 n+\alpha+\beta+2}\left[(n+\alpha+1) J_{n}^{\alpha, \beta}(x)-(n+1) J_{n+1}^{\alpha, \beta}(x)\right]
\end{aligned}
$$




$$
\begin{aligned}
& (1+x) J_{n}^{\alpha, \beta+1}(x)=\frac{2}{2 n+\alpha+\beta+2}\left[(n+\beta+1) J_{n}^{\alpha, \beta}(x)+(n+1) J_{n+1}^{\alpha, \beta}(x)\right] ; \\
& \partial_{x} J_{n}^{\alpha, \beta}(x)=\frac{1}{2}(n+\alpha+\beta+1) J_{n-1}^{\alpha+1, \beta+1}(x), \quad n \geqslant 1 ; \\
& \omega^{\alpha, \beta}(x) J_{n}^{\alpha, \beta}(x)=\frac{(-1)^{m}(n-m) !}{2^{m} n !} \frac{d^{m}}{d x^{m}}\left\{\omega^{\alpha+m, \beta+m}(x) J_{n-m}^{\alpha+m, \beta+m}(x)\right\}, \quad n \geqslant m \geqslant 0 ; \\
& x J_{n}^{\alpha, \beta}(x)=a_{n} J_{n-1}^{\alpha, \beta}(x)+b_{n} J_{n}^{\alpha, \beta}(x)+c_{n} J_{n+1}^{\alpha, \beta}(x),
\end{aligned}
$$

where $a_{n}, b_{n}, c_{n}$ are constants (see [35] for their expressions).

\section{Appendix B. Some proofs}

\section{B.1. The proof of (2.10)}

We first consider the case $(\alpha, \beta) \in \aleph_{1}$. Taking $Y_{n_{1}}(x)=\omega^{-\alpha,-\beta}(x) J_{n_{1}}^{-\alpha,-\beta}(x)=j_{n}^{\alpha, \beta}(x)$ in (A.2), we find that

$$
\left(1-x^{2}\right) \partial_{x}^{2} j_{n}^{\alpha, \beta}(x)+[(\beta-\alpha)-(\alpha+\beta+2) x] \partial_{x} j_{n}^{\alpha, \beta}(x)+\lambda_{n}^{\alpha, \beta} j_{n}^{\alpha, \beta}(x)=0 .
$$

Multiplying $\omega^{\alpha, \beta}(x)$ on both sides of the above equation, we can rewrite the resulting equation as (2.10) with $\lambda_{n}^{\alpha, \beta}=$ $\left(n_{1}+1\right)\left(n_{1}-\alpha-\beta\right)$.

Next, let $(\alpha, \beta) \in \aleph_{2}$. By the definition (2.7), we have $J_{n_{1}}^{-\alpha, \beta}(x)=(1-x)^{\alpha} j_{n}^{\alpha, \beta}(x)$. We plug it into (A.1) to get that

$$
\partial_{x}\left((1-x)^{-\alpha+1}(1+x)^{\beta+1} \partial_{x}\left((1-x)^{\alpha} j_{n}^{\alpha, \beta}(x)\right)\right)+\mu_{n_{1}}^{-\alpha, \beta}(1+x)^{\beta} j_{n}^{\alpha, \beta}(x)=0,
$$

which can be simplified to

$$
\left(1-x^{2}\right) \partial_{x}^{2} j_{n}^{\alpha, \beta}(x)+[(\beta-\alpha)-(\alpha+\beta+2) x] \partial_{x} j_{n}^{\alpha, \beta}(x)+\left(\mu_{n_{1}}^{-\alpha, \beta}-\alpha(\beta+1)\right) j_{n}^{\alpha, \beta}(x)=0 .
$$

Multiplying $\omega^{\alpha, \beta}(x)$ on both sides of the above equation, we can get the resulting equation (2.10) with $(\alpha, \beta) \in \aleph_{2}$. We can prove the case $(\alpha, \beta) \in \aleph_{3}$ similarly.

Finally (2.10) with $(\alpha, \beta) \in \aleph_{4}$ is a direct consequence of (A.1) and (2.7).

\section{B.2. The proof of Lemma 2.1}

We first prove (2.16). For $\alpha, \beta \leqslant-2$, let $n_{1}=n-[-\alpha]-[-\beta] \geqslant 0$, and by (A.9) and (2.7),

$$
\begin{aligned}
j_{n-1}^{\alpha+1, \beta+1}(x) & \stackrel{(2.7)}{=}(1-x)^{-\alpha-1}(1+x)^{-\beta-1} J_{n_{1}+1}^{-\alpha-1,-\beta-1}(x) \\
& \stackrel{(\text { A.9) }}{=}-\frac{1}{2\left(n_{1}+1\right)} \partial_{x}\left((1-x)^{-\alpha}(1+x)^{-\beta} J_{n_{1}}^{-\alpha,-\beta}(x)\right) \\
& \stackrel{(2.7)}{=}-\frac{1}{2(n-[-\alpha]-[-\beta]+1)} \partial_{x} j_{n}^{\alpha, \beta}(x) .
\end{aligned}
$$

This leads to (2.16) for the case (i) of the condition (2.15). In fact, (B.1) also holds for $\alpha=-1$ and $\beta \leqslant-2$, which, along with (2.7) (the cases $(-1, \beta) \in \aleph_{1}$ and $\left.(0, \beta+1) \in \aleph_{3}\right)$, leads to

$$
\begin{aligned}
j_{n-1}^{0, \beta+1}(x) & =(1+x)^{-\beta-1} J_{n_{1}+1}^{0,-\beta-1}(x)=-\frac{1}{2\left(n_{1}+1\right)} \partial_{x}\left(\omega^{1,-\beta}(x) J_{n_{1}}^{1,-\beta}(x)\right) \\
& =-\frac{1}{2\left(n_{1}+1\right)} \partial_{x} j_{n}^{-1, \beta}(x), \quad n_{1}=n-[-\beta]-1 \geqslant 0 .
\end{aligned}
$$

Hence, (2.16) holds for the case (ii) of the condition (2.15). Similarly, we can prove the case: $\alpha \leqslant-2$ and $\beta=-1$, while taking $\alpha=\beta=-1$ in (B.1) gives (2.16) for the case (iv) of the condition (2.15).

We now turn to the proof of (2.18). If $\alpha \leqslant-2$ and $\beta>-1$, then, using (A.6), (A.8) and (2.7) with $n_{1}=n-[-\alpha]$, yields that for $n_{1} \geqslant 0$, 


$$
\begin{aligned}
\partial_{x} j_{n}^{\alpha, \beta}(x) & \stackrel{(2.7)}{=} \partial_{x}\left((1-x)^{-\alpha} J_{n_{1}}^{-\alpha, \beta}(x)\right) \\
& =(1-x)^{-\alpha-1}\left(\alpha J_{n_{1}}^{-\alpha, \beta}(x)+(1-x) \partial_{x} J_{n_{1}}^{-\alpha, \beta}(x)\right) \\
& \stackrel{(\text { A. } 8)}{=}(1-x)^{-\alpha-1}\left(\alpha J_{n_{1}}^{-\alpha, \beta}(x)+\frac{1}{2}\left(n_{1}-\alpha+\beta+1\right)(1-x) J_{n_{1}-1}^{-\alpha+1, \beta+1}(x)\right) \\
& \stackrel{(\text { A.6) }}{=}(1-x)^{-\alpha-1}\left(\alpha J_{n_{1}}^{-\alpha, \beta}(x)+\frac{n_{1}-\alpha+\beta+1}{2 n_{1}-\alpha+\beta+1}\left(\left(n_{1}-\alpha\right) J_{n_{1}-1}^{-\alpha, \beta+1}(x)-n_{1} J_{n_{1}}^{-\alpha, \beta+1}(x)\right)\right) .
\end{aligned}
$$

Using (A.4) gives

$$
J_{n_{1}-1}^{-\alpha, \beta+1}(x)=J_{n_{1}}^{-\alpha, \beta}(x)-J_{n_{1}}^{-\alpha-1, \beta+1}(x),
$$

and plugging it into the above formula leads to

$$
\begin{aligned}
\partial_{x} j_{n}^{\alpha, \beta}(x)= & (1-x)^{-\alpha-1}\left\{\alpha J_{n_{1}}^{-\alpha, \beta}(x)+\frac{n_{1}-\alpha+\beta+1}{2 n_{1}-\alpha+\beta+1}\left(\left(n_{1}-\alpha\right) J_{n_{1}}^{-\alpha, \beta}(x)\right.\right. \\
& \left.\left.-\left(n_{1}-\alpha\right) J_{n_{1}}^{-\alpha-1, \beta+1}(x)-n_{1} J_{n_{1}}^{-\alpha, \beta+1}(x)\right)\right\} \\
= & (1-x)^{-\alpha-1}\left\{\frac{n_{1}}{2 n_{1}-\alpha+\beta+1}\left(\left(n_{1}+\beta+1\right) J_{n_{1}}^{-\alpha, \beta}(x)-\left(n_{1}-\alpha+\beta+1\right) J_{n_{1}}^{-\alpha, \beta+1}(x)\right)\right. \\
& \left.-\frac{\left(n_{1}-\alpha\right)\left(n_{1}-\alpha+\beta+1\right)}{2 n_{1}-\alpha+\beta+1} J_{n_{1}}^{-\alpha-1, \beta+1}(x)\right\} .
\end{aligned}
$$

Thanks to (A.5), we have

$$
\left(n_{1}-\alpha+\beta+1\right) J_{n_{1}}^{-\alpha, \beta+1}(x)=\left(n_{1}+\beta+1\right) J_{n_{1}}^{-\alpha, \beta}(x)+\left(n_{1}-\alpha\right) J_{n_{1}}^{-\alpha-1, \beta+1}(x) .
$$

Consequently,

$$
\begin{aligned}
\partial_{x} j_{n}^{\alpha, \beta}(x) & =(1-x)^{-\alpha-1}\left(\frac{-n_{1}\left(n_{1}-\alpha\right)}{2 n_{1}-\alpha+\beta+1} J_{n_{1}}^{-\alpha-1, \beta+1}(x)-\frac{\left(n_{1}-\alpha\right)\left(n_{1}-\alpha+\beta+1\right)}{2 n_{1}-\alpha+\beta+1} J_{n_{1}}^{-\alpha-1, \beta+1}(x)\right) \\
& =-\left(n_{1}-\alpha\right)(1-x)^{-\alpha-1} J_{n_{1}}^{-\alpha-1, \beta+1}(x) \\
& \stackrel{(2.7)}{=}-(n-[-\alpha]-\alpha) j_{n-1}^{\alpha+1, \beta+1}(x) .
\end{aligned}
$$

Hence, (2.18) holds for the case (i) of condition (2.17). Note that the above procedure is also valid for $\alpha=-1$ and $\beta>-1$, namely,

$$
\partial_{x} j_{n}^{-1, \beta}(x)=-n J_{n-1}^{0, \beta+1}(x)=-n j_{n-1}^{0, \beta+1}(x), \quad n \geqslant 1 .
$$

Here, we used the definition (2.7) (with $\left.(0, \beta+1) \in \aleph_{4}\right)$ to derive the last identity. This implies (2.18) for the case (ii) of condition (2.17).

Finally, (2.20) can be verified by using the property (2.13) and (2.18).

\section{B.3. The proof of Lemma 2.2}

We first prove (2.21).

If $m \leqslant k$, then we know from the condition (2.17) that the derivative relation (2.18) is valid for $\alpha=-k \leqslant-1$ and $\beta>-1$, and using it inductively leads to the desired result (2.21) in case of $m \leqslant k$.

Next, thanks to (A.8), we derive that for $a, b>-1$,

$$
\partial_{x}^{p} J_{q}^{a, b}(x)=\frac{\Gamma(q+p+a+b+1)}{2^{p} \Gamma(q+a+b+1)} J_{q-p}^{a+p, b+p}(x), \quad q \geqslant p, p, q \in \mathbb{N} .
$$

Thus, for $m>k$, we deduce from (2.18) with $m=k$ and the above formula that 


$$
\begin{aligned}
\partial_{x}^{m} j_{n}^{-k, \beta}(x) & =\partial_{x}^{m-k} \partial_{x}^{k} j_{n}^{-k, \beta}(x) \\
& \stackrel{(2.21)}{=}(-1)^{k}\left(\prod_{i=0}^{k-1}(n-i)\right) \partial_{x}^{m-k} j_{n-k}^{0, \beta+k}(x) \\
& \stackrel{(2.7)}{=}(-1)^{k}\left(\prod_{i=0}^{k-1}(n-i)\right) \partial_{x}^{m-k} J_{n-k}^{0, \beta+k}(x) \\
& \stackrel{(\text { B.3) }}{=}(-1)^{k}\left(\prod_{i=0}^{k-1}(n-i)\right) \frac{\Gamma(n+m-k+\beta+1)}{2^{m-k} \Gamma(n+\beta+1)} J_{n-m}^{m-k, \beta+m}(x) \\
& \stackrel{(2.7)}{=} D_{m, n}^{k, \beta} j_{n-m}^{-k+m, \beta+m}(x) .
\end{aligned}
$$

We used the definition (2.7) with $(-k+m, \beta+m) \in \aleph_{4}$ to derive the last identity.

The result (2.23) follows from (2.13) and (2.21)-(2.22).

We now turn to the proof of (2.24). For the first case: $m \leqslant l \leqslant k$, we can derive the result by using (2.16) inductively. For the second case: $l<m \leqslant k$, we use the above result with $m=l, l<k$ and (2.21) with $m-l \leqslant k-l$, to deduce that

$$
\begin{aligned}
\partial_{x}^{m} j_{n}^{-k,-l}(x) & =\partial_{x}^{m-l} \partial_{x}^{l} j_{n}^{-k,-l}(x)=(-2)^{l}\left(\prod_{i=1}^{l}(n-l-k+i)\right) \partial_{x}^{m-l} j_{n-l}^{-k+l, 0}(x) \\
& =(-2)^{l}\left(\prod_{i=1}^{l}(n-l-k+i)\right)\left((-1)^{m-l} \prod_{i=0}^{m-l-1}(n-l-i)\right) j_{n-m}^{-k+m, m-l}(x) \\
& =E_{m, n}^{k, l} j_{n-m}^{-k+m,-l+m}(x) .
\end{aligned}
$$

We can prove the result with $l<k \leqslant m$ in the same manner. Finally, the result (2.26) follows from (2.25) and (2.13).

\section{B.4. Derivation of (3.1)-(3.4)}

Let $\alpha, \beta<1$ and $n_{1}=n-[-\alpha]-[-\beta]$. By the definition (2.7) and (A.6), (A.7),

$$
\begin{aligned}
& j_{n}^{\alpha-1, \beta}(x)=\frac{2}{2 n_{1}-\alpha-\beta}\left[\left(n_{1}-\alpha\right) j_{n-1}^{\alpha, \beta}(x)-n_{1} j_{n}^{\alpha, \beta}(x)\right], \\
& j_{n}^{\alpha, \beta-1}(x)=\frac{2}{2 n_{1}-\alpha-\beta}\left[\left(n_{1}-\alpha\right) j_{n-1}^{\alpha, \beta}(x)+n_{1} j_{n}^{\alpha, \beta}(x)\right] .
\end{aligned}
$$

Hence, taking $\alpha=\beta=0$ leads to

$$
j_{n}^{-1,0}(x)=L_{n-1}(x)-L_{n}(x), \quad j_{n}^{0,-1}(x)=L_{n-1}(x)+L_{n}(x) .
$$

Next, we verify from (A.6), (A.7) that for $a, b>-1$,

$$
\left(1-x^{2}\right) J_{k-1}^{a+1, b+1}(x)=A_{k}^{a, b} J_{k-1}^{a, b}(x)+B_{k}^{a, b} J_{k}^{a, b}(x)+C_{k}^{a, b} J_{k+1}^{a, b}(x),
$$

where

$$
\begin{aligned}
A_{k}^{a, b} & =\frac{4(k+a)(k+b)}{(2 k+a+b)(2 k+a+b+1)}, \quad B_{k}^{a, b}=\frac{4 k(a-b)}{(2 k+a+b)(2 k+a+b+2)}, \\
C_{k}^{a, b} & =\frac{4 k(k+1)}{(2 k+a+b+1)(2 k+a+b+2)} .
\end{aligned}
$$

Taking $a=-\alpha, b=-\beta$ and $k=n_{1}$, we derive from (B.6), (B.7) and the definition (2.7) that

$$
j_{n+1}^{\alpha-1, \beta-1}(x)=A_{n_{1}}^{-\alpha,-\beta} j_{n-1}^{\alpha, \beta}(x)+B_{n_{1}}^{-\alpha,-\beta} j_{n}^{\alpha, \beta}(x)+C_{n_{1}}^{-\alpha,-\beta} j_{n+1}^{\alpha, \beta}(x) .
$$


Thus, we have

$$
j_{n+1}^{-1,-1}(x)=\frac{2 n}{2 n+1}\left(L_{n-1}(x)-L_{n+1}(x)\right),
$$

which implies (3.1). Similarly, taking $(\alpha, \beta)=(-1,0),(0,-1),(-1,-1)$ in (B.8), and using (B.5) and/or (B.9), we derive (3.2)-(3.4).

\section{References}

[1] R. Agarwal, Boundary Value Problems for Higher Ordinary Differential Equations, World Scientific, Singapore, 1986.

[2] R. Askey, Orthogonal Polynomials and Special Functions, Society for Industrial and Applied Mathematics, Philadelphia, PA, 1975.

[3] I. Babuška, B. Guo, Optimal estimates for lower and upper bounds of approximation errors in the $p$-version of the finite element method in two dimensions, Numer. Math. 85 (2) (2000) 219-255.

[4] I. Babuška, B. Guo, Direct and inverse approximation theorems for the p-version of the finite element method in the framework of weighted Besov spaces. I. Approximability of functions in the weighted Besov spaces, SIAM J. Numer. Anal. 39 (5) (2001/2002) 1512-1538 (electronic).

[5] J. Bergh, J. Löfström, Interpolation Spaces, An Introduction, Springer-Verlag, Berlin, 1976.

[6] C. Bernardi, M. Dauge, Y. Maday, Spectral Methods for Axisymmetric Domains, Gauthier-Villars, Éditions Scientifiques et Médicales Elsevier, Paris, 1999.

[7] C. Bernardi, Y. Maday, Approximations Spectrales de Problèmes aux Limites Elliptiques, Springer-Verlag, Paris, 1992.

[8] C. Bernardi, Y. Maday, Basic results on spectral methods. R94022, Univ. Pierre et Marie Curie, Paris, 1994.

[9] C. Bernardi, Y. Maday, Spectral method, Part 2, in: P.G. Ciarlet, L.L. Lions (Eds.), Handbook of Numerical Analysis, vol. 5, North-Holland, Amsterdam, The Netherlands, 1997.

[10] J.L. Bona, V.A. Dougalis, O.A. Karakashian, Conservative, high-order numerical schemes for the generalized KdV-type equation, Philos Trans. Roy. Soc. Lond. Ser. A 351 (1995) 107-164.

[11] A. Boutayeb, E. Twizell, Numerical methods for the solution of special sixth-order boundary value problems, Int. J. Comput. Math. 50 (1992) 207-233.

[12] J.P. Boyd, Chebyshev and Fourier Spectral Methods, second ed., Dover Publications Inc., Mineola, NY, 2001.

[13] C. Canuto, M.Y. Hussaini, A. Quarteroni, T.A. Zang, Spectral Methods: Fundamentals in Single Domains, Springer-Verlag, Heidelberg, 2006

[14] C. Canuto, A. Quarteroni, Approximation results for orthogonal polynomials in Sobolev spaces, Math. Comp. 38 (1982) 67-86.

[15] R. Courant, D. Hilbert, Methods of Mathematical Physics. Volume 1, Interscience Publishers, New York, 1953.

[16] B. Dey, A. Khare, C.N. Kumar, Stationary solitons of the fifth order KdV-type equations and their stability, Phys. Lett. A 223 (1996) 449-452

[17] M. El-Gamel, J.R. Cannon, A.I. Zayed, Sinc-Galerkin method for solving linear sixth-order boundary value problems, Math. Comp. 73 (2004) $1-19$.

[18] B. Fornberg, A pseudospectral fictitious point method for high order initial-boundary value problems, SIAM J. Sci. Comput. 28 (5) (2006) 1716-1729 (electronic).

[19] D. Funaro, Polynomial Approximations of Differential Equations, Springer-Verlag, Berlin, 1992.

[20] D. Gottlieb, S.A. Orszag, Numerical Analysis of Spectral Methods: Theory and Applications, SIAM-CBMS, Philadelphia, 1977.

[21] B. Guo, Gegenbauer approximation and its applications to differential equations on the whole line, J. Math. Anal. Appl. 226 (1998) 180-206.

[22] B. Guo, Spectral Methods and their Applications, World Scientific Publishing Co. Inc., River Edge, NJ, 1998.

[23] B. Guo, Jacobi approximations in certain Hilbert spaces and their applications to singular differential equations, J. Math. Anal. Appl. 243 (2000) 373-408.

[24] B. Guo, Jacobi spectral approximation and its applications to differential equations on the half line, J. Comput. Math. 18 (2000) 95-112.

[25] B. Guo, J. Shen, L.-L. Wang, Optimal spectral-Galerkin methods using generalized Jacobi polynomials, J. Sci. Comp. 27 (2006) $305-322$.

[26] B. Guo, L. Wang, Jacobi approximations and Jacobi-Gauss-type interpolations in nonuniformly weighted Sobolev spaces, J. Approx. Theory $128(2004) 1-41$.

[27] W.Z. Huang, D.M. Sloan, The pseudospectral method for third-order differential equations, SIAM J. Numer. Anal. 29 (6) (1992) $1626-1647$.

[28] S. Kichenassamy, P.J. Olver, Existence and nonexistence of solitary wave solutions to higher-order model evolution equations, SIAM J. Math. Anal. 23 (5) (1992) 1141-1166.

[29] W.J. Merryfield, B. Shizgal, Properties of collocation third-derivative operators, J. Comput. Phys. 105 (1) (1993) $182-185$.

[30] E.J. Parkes, Z. Zhu, B.R. Duffy, H.C. Huang, Sech-polynomial travelling solitary-wave solutions of odd-order generalized KdV-type equations, Phys. Lett. A 248 (1998) 219-224.

[31] E.D. Rainville, Special Functions, Macmillan, New York, 1960.

[32] J. Shen, Efficient spectral-Galerkin method I. direct solvers for second- and fourth-order equations by using Legendre polynomials, SIAM J. Sci. Comput. 15 (1994) 1489-1505.

[33] J. Shen, A new dual-Petrov-Galerkin method for third and higher odd-order differential equations: application to the KDV equation, SIAM J. Numer. Anal. 41 (2003) 1595-1619.

[34] J. Shen, L.-L. Wang, Legendre and Chebyshev dual-Petrov-Galerkin methods for hyperbolic equations, Comput. Methods Appl. Mech. Engrg. 196 (37-40) (2007) 3785-3797.

[35] G. Szegö, Orthogonal Polynomials, fourth ed., Amer. Math. Soc. Collog. Publ., vol. 23, Amer. Math. Soc., Providence, RI, 1975.

[36] A.F. Timan, Theory of Approximation of Functions of a Real Variable, Pergamon, Oxford, 1963.

[37] N.J. Zabusky, C.J. Galvin, Shallow water waves, the Korteveg-de-Vries equation and solitons, J. Fluid Mech. 47 (1971) $811-824$. 